\title{
Modelling of Dispersive PT-Symmetric Bragg Grating
}

\author{
Sendy Phang*", Ana Vukovic*, Hadi Susanto ${ }^{\dagger}$, Trevor M. Benson*, Phillip Sewell* \\ ${ }^{\star}$ The George Green Institute for Electromagnetics Research,University of Nottingham, UK, NG7 2RD, \\ 1eexsp14@nottingham.ac.uk \\ ${ }^{+}$School of Mathematical Sciences, University of Nottingham, UK, NG7 2RD
}

Keywords: transmission line modelling method, dispersive material, gain saturation.

\begin{abstract}
This paper reports on the time-domain numerical model of a parity-time Bragg grating with saturated and dispersive gain. The model is compared against the ideal PT scenario where the gain is constant and unsaturated for all frequencies.
\end{abstract}

\section{Introduction}

Recently, a new class of optical metamaterials that utilise balanced loss and gain, also known as Parity-Time (PT) structures, have opened a new avenue in realising optical functionalities, for example, switching [1][2], lasing and isolating. An interesting potential application of PT devices is in cloaking as they offer unidirectional invisibility over a wide frequency range [3].

To date, all modelling of PT-devices has been done using frequency domain methods such as coupled mode theory and using materials that have constant and frequency-independent gain and loss. However, in practice the gain in material saturates at high input intensities and any change in the real or imaginary part in the material refractive index is described via the Kramers-Kronig relations.

This paper outlines the implementation of a dispersive gain material model that satisfies the Kramers-Kronig relation and gain saturation. This is done in the numerical Transmission Line Modelling (TLM) method. The model is then used to analyse the response of a PT Bragg grating and compare it with the idealised case with constant gain/loss.

\section{PT-Symmetric Bragg grating}

A schematic presentation of a PT Bragg grating is given in Fig. 1 . The length of the period is $\Lambda=\lambda_{B} /\left(2 n_{R}\right)$, where $\lambda_{B}$ is the Bragg wavelength and $n_{R}$ is the average refractive index of the grating. The PT- structure requires that loss and gain in the structure are balanced which can be expressed via a complex refractive index as $\hat{n}(-z)=\hat{n}^{*}(z)$. This condition implies that one period of the Bragg grating has four layers of equal length but of different material parameters, so that refractive indices of layers satisfy the following distribution:

$$
\left\{\begin{array}{c}
n_{R}+\Delta n_{R}+\text { gain }, \quad 0<x<\Lambda / 4 \\
n_{R}-\Delta n_{R}+\text { gain, } \Lambda / 4<x<\Lambda / 2 \\
n_{R}-\Delta n_{R}+\text { loss }, \quad \Lambda / 2<x<3 \Lambda / 4 \\
n_{R}+\Delta n_{R}+\text { loss }, \quad 3 \Lambda / 4<x<\Lambda
\end{array}\right.
$$

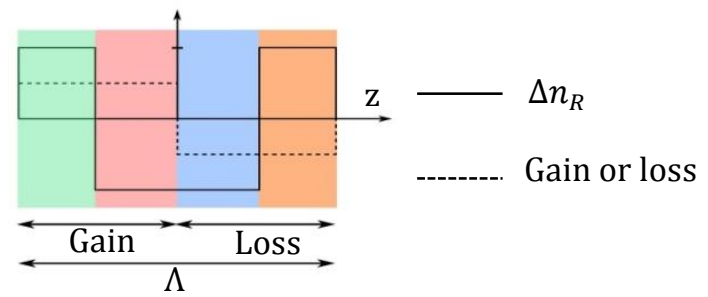

Fig. 1. Schematic illustration for a single period of the PTBragg grating

\section{Modelling}

In this paper, a model of macroscopic physical gain and loss, having a homogenously-spectral-broadening and satisfying the Kramers-Kronig condition [4] is implemented through the electric conductivity $\sigma$, as

$\sigma(\omega)=\left(\frac{1}{1+I / I_{s}}\right)\left(\frac{\sigma_{0} / 2}{1+j\left(\omega-\omega_{0 \sigma}\right) \tau}+\frac{\sigma_{0} / 2}{1+j\left(\omega+\omega_{0 \sigma}\right) \tau}\right)$

where $\omega_{0 \sigma}$ is the atomic transition angular-frequency, $\sigma_{0} / 2$ is the peak value of the conductivity at $\omega_{0 \sigma}$ and $\tau$ is the dipole relaxation time parameter. Material gain and loss are implemented as

$$
\alpha(\omega)= \pm \frac{\sigma(\omega)}{2 c n_{R} \epsilon_{0}}
$$

where $c$ and $\epsilon_{0}$ are the free-space light velocity and permittivity respectively, and $n_{R}$ is the real part of refractive index. Loss is implemented with a positive sign whilst gain is implemented with a negative sign in eq. (3).

The frequency dependant dielectric properties are modelled using the Duffing model for electric polarization outlined in [5] as,

$$
\frac{\partial^{2} P_{y D}}{\partial t^{2}}+2 \delta \frac{\partial P_{y D}}{\partial t}+\omega_{0}^{2} P_{y D}=\epsilon_{0} \chi_{e 0} \omega_{0 D}^{2} E_{y}
$$

where $P_{y D}$ and $E_{y}$ denote the electric polarization and field in the $y$-direction, $\delta$ and $\omega_{0 D}$ denote the damping and dielectric resonant angular-frequency parameters of the medium, and $\chi_{e 0}$ denotes the dielectric susceptibility at DC frequency.

The implementation of the gain (2) and Duffing (4) models are done in the Transmission Line Modelling method [6]. The TLM method is a time stepping numerical technique based upon the analogy between the propagating electromagnetic fields and voltage impulses travelling on an interconnected mesh of transmission lines. As a time-domain numerical model, it offers flexibility in modelling frequencydependent and nonlinear material [6]. In the TLM method, this is done using $z$-transform approach. The overall outline of the TLM approach in modelling dispersive properties is to 
first calculate the electric field at each node from the incident voltages from the left $V_{L}^{i}$ and right $V_{R}^{i}$ nodes. For the 1D case this is given by [6],

$$
2\left(V_{L}^{i}+V_{R}^{i}\right)=2 V_{y}+g(z) V_{y}+2\left(\frac{1-z^{-1}}{1+z^{-1}}\right)\left(\chi_{e \infty} V_{y}+p_{y D}\right)
$$

where $V_{y}$ is the normalized electric field in the $y$-direction, $\chi_{e \infty}$ is the dielectric susceptibility at infinite frequency, $g(z)$ and $p_{y D}(z)$ are the normalized conductivity (2) and electric polarization (4) in the $z$-domain. In the next step the scattered voltages from the each node are obtained which become the incident voltages on the neighbouring nodes in the following time-step. These successive repetitions of the scatterpropagate procedure provide an explicit and stable time stepping algorithm that mimics electromagnetic field behaviour to second order accuracy in both time and space.

\section{Result and discussions}

In this section, a PT Bragg grating based on GaAs material is analysed. The material parameters chosen are: $\chi_{e 0}=7.5$, $\omega_{O D}=4614.4 \mathrm{rad} / \mathrm{ps}$, and $\delta=0.0923 \mathrm{rad} / \mathrm{ps}$ [7] with the high and low dielectric susceptibility as $\chi_{e \infty H}=2.8$ and $\chi_{e \infty L}=2.5$. The gain parameters are $I_{s}=65.2 \mathrm{~kW} / \mathrm{cm}^{2}$, $\tau=0.1 \mathrm{ps}$ and $\omega_{0 \sigma}=1883 \mathrm{rad} / \mathrm{ps}$ [4]. The period of the Bragg grating is designed so that the band-gap is centred at the atomic-transition angular frequency $\omega_{0 \sigma}$, i.e. $\Lambda=$ $0.14058 \mu \mathrm{m}$. The background material has $n_{R}=3.56$, i.e. the refractive index of GaAs at around $1 \mu \mathrm{m}$.

Figure 2 compares the transmittance of the 200 period GaAs PT Bragg grating for the ideal case, the physical model and the linear grating that corresponds to the conventional Bragg grating with no gain/loss. The ideal PT model has a constant and frequency-independent gain with $\alpha=$ $0.1325 \mu \mathrm{m}^{-1}$ with and modulation index of $\Delta n_{R}=0.0211$. The physical gain model has a parameter of $\alpha\left(\omega_{0 \sigma}\right)=$ $0.1325 \mu \mathrm{m}^{-1}$ and was run for low $\left(I=0.05 \mathrm{~kW} / \mathrm{cm}^{2}\right)$ and high intensity $\left(I=50 \mathrm{~kW} / \mathrm{cm}^{2}\right)$. The linear Bragg grating has $\alpha=0$ and is included for reference. Figures 2(a,b) show the transmittance and the reflectance for a wave incident from the right respectively. Figures 2(a,b) show that the PT Bragg grating ideal model exhibits a broadband unidirectional invisibility i.e. $T_{L}=T_{R} \approx 1$ and $\Gamma_{R}=0$. However, in the case of the physical model unidirectional invisibility occurs only around the band gap frequency, and is dependent on the input signal intensity, reducing the unidirectional invisibility to the narrowband range around the Bragg frequency. The effect is more prominent at high input intensities which can be explained by the fact that the saturation of the gain breaks the balance of gain and loss thereby destroying the PT-symmetry.

\section{Conclusion}

The paper reports on the implementation of dispersive and saturated gain model in the TLM for the time-domain modelling of the unidirectional invisibility of the PTsymmetric Bragg gratings. The saturated gain modifies the unidirectional invisibility of the grating making it dependent on the intensity of the input signal. The model shows that the realistic model of gain modifies the unidirectional invisibility of the grating reducing it to a narrowband phenomenon.
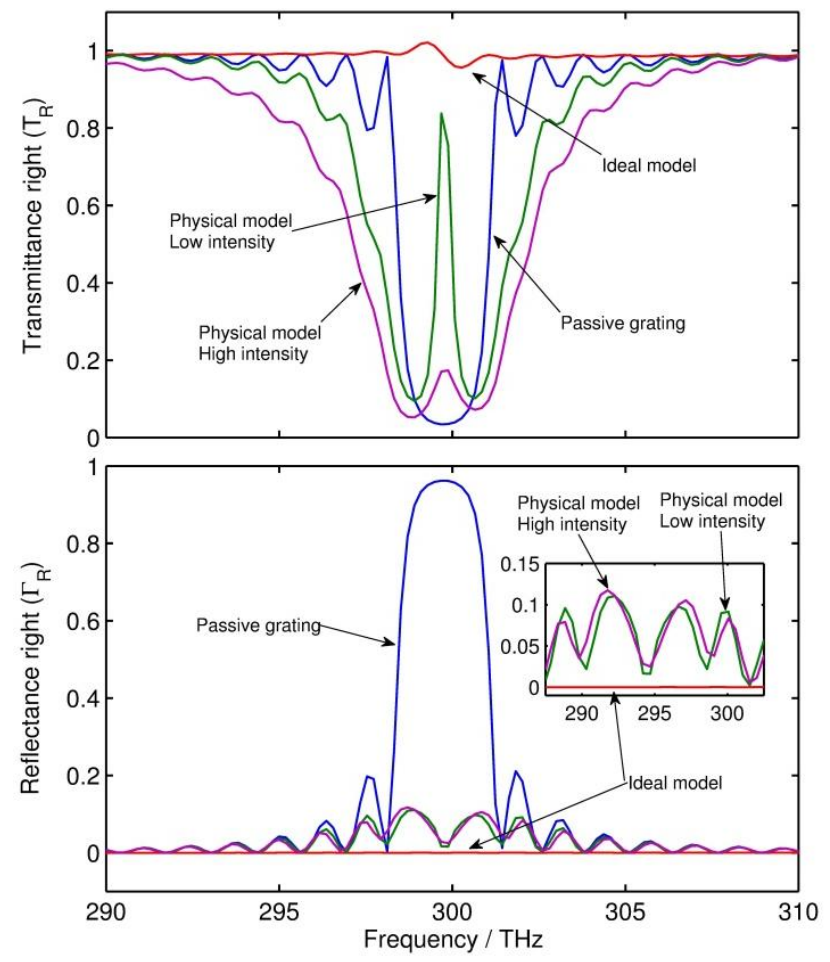

Fig. 2. Plot of dispersion of PTBG for passive Bragg filter, PTBG with the ideal model, physical model (low and high intensity) for incident from the right.

\section{References}

[1] F. Nazari, M. Nazari, and M. K. Moravvej-Farshi, "A $2 \times 2$ spatial optical switch based on PT-symmetry.," Opt. Lett., vol. 36, no. 22, pp. 4368-70, Nov. 2011.

[2] S. Phang, A. Vukovic, H. Susanto, T. M. Benson, and P. Sewell, "Ultrafast optical switching using paritytime symmetric Bragg gratings," J. Opt. Soc. Am. B, vol. 30, no. 11, p. 2984, Oct. 2013.

[3] Z. Lin, H. Ramezani, T. Eichelkraut, T. Kottos, H. Cao, and D. N. Christodoulides, "Unidirectional Invisibility Induced by PT-Symmetric Periodic Structures," Phys. Rev. Lett., vol. 106, no. 21, p. 213901, May 2011.

[4] S. C. Hagness, R. M. Joseph, and A. Taflove, "Subpicosecond electrodynamics of distributed Bragg reflector microlasers: Results from finite difference time domain simulations," Radio Sci., vol. 31, no. 4, pp. 931-941, Jul. 1996.

[5] V. Janyani, A. Vukovic, and J. Paul, "The development of TLM models for nonlinear optics," Mikrotalasna Rev., vol. 10, no. 1, pp. 35-42, 2004.

[6] J. Paul, "Modelling of general electromagnetic material properties in TLM," University of Nottingham, 1998.

[7] M. Bass, G. Li, and E. van Stryland, Handbook of optics vol. 4, 3rd ed. New York, NY: McGraw Hill, 2010. 